\title{
MODELING AND CONTROL OF A 3-DEGREE-OF-FREEDOM WALKING MICROROBOT
}

\author{
Yu-Ming Chen ${ }^{1}$, John W. Suh ${ }^{2}$, Gregory T. A. Kovacs ${ }^{2}$, R. Bruce Darling ${ }^{1}$, Karl F. Böhringer ${ }^{1}$ \\ ${ }^{1}$ University of Washington, Seattle, Washington, USA \\ ${ }^{2}$ Stanford University, Stanford, CA, USA
}

\begin{abstract}
This paper describes a walking microrobot with 512 thermobimorph polyimide actuator legs. Eight groups of unidirectional legs can be separately controlled to produce planar motion with three degrees of freedom. Wave-like gaits propel the robot at speeds of up to $250 \mu \mathrm{m} / \mathrm{sec}$ while consuming approximately $1.6 \mathrm{~W}$ power. Finite element methods are employed to investigate the force generation and the thermal characteristics of the robot. Results from simulation and experiments lead to a compact model for the relationship between temperature and velocity of the robot.
\end{abstract}
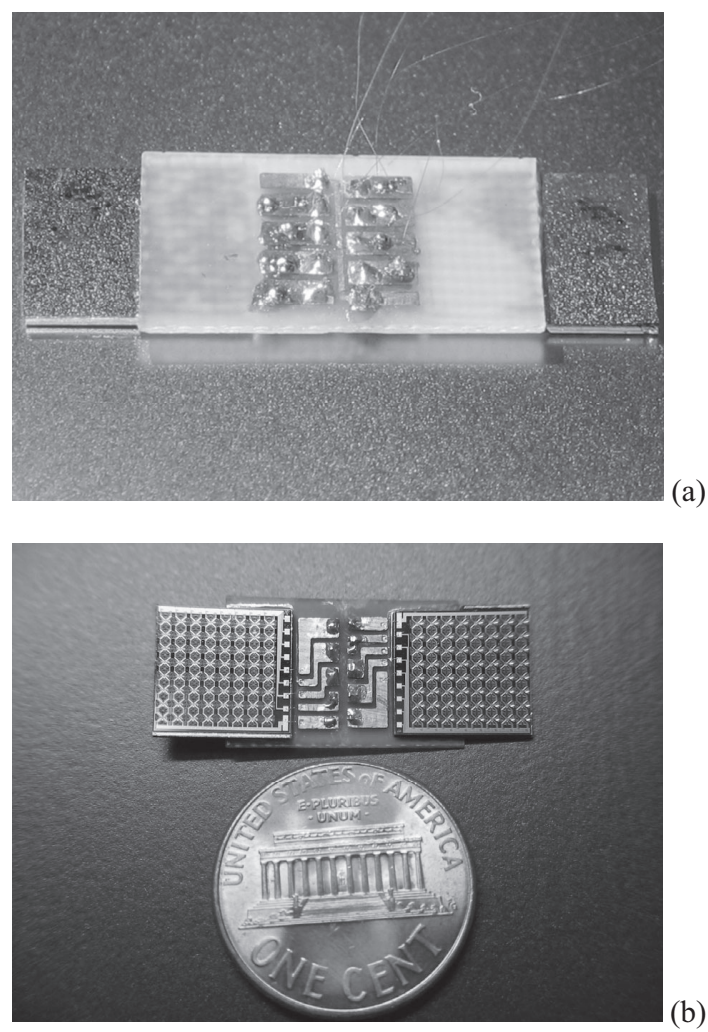

Figure 1. (a) Top and (b) bottom view of the microrobot. The two cilia chips can be seen attached and wire bonded to a PCB backbone. Each chip consists of an $8 \times 8$ array of "motion pixels" (i.e., a group of 4 orthogonal cilia). The robot measures approximately $3 \mathrm{~cm}$ length, $1 \mathrm{~cm}$ width and $0.5 \mathrm{~mm}$ thickness.

\section{INTRODUCTION}

Microrobots offer novel solutions for inspection and maintenance of equipment in otherwise inaccessible places, searchand-rescue, reconnaissance, and possibly self-organizing structures. Analogies to ants, grasshoppers, and waterbugs have been invoked in prior work on walking microrobots, e.g. [1-4]. This paper summarizes the experience gathered from an insect-like walking microrobot, including modeling and simulation of its actuators, control strategies for actuator gaits, and several years of experiments under a variety of operating conditions.

\section{SYSTEM ARCHITECTURE}

Our system, previously introduced in a preliminary conference abstract [5], consists of two arrays of thermo-bimorph ciliary actuator arrays connected by a small PCB "backbone" (Fig. 1). Each actuator chip measures $1.1 \mathrm{~mm}$ squared and supports an $8 \times 8$ array of "motion pixels" consisting of 4 cilia-like thermobimorph actuators capable of generating force with in-plane components in 4 orthogonal directions when activated by an internal resistive heater (Fig. 2). Two layers of polyimide with widely disparate coefficients of thermal expansion (CTE) are the main structural components of the cilia. Since the CTE of the top layer is larger than the CTE of the bottom layer, they curl out of the substrate plane in their cool, inactive state due to internal thermal stress induced by the initial curing at $350^{\circ} \mathrm{C}$. As the temperature is increased towards $350^{\circ} \mathrm{C}$, the radius of curvature increases and the cilia flatten out towards the substrate surface. Fabrication of the cilia was discussed in detail in [6].

The motion pixels are addressed via 8 control wires and 1 ground wire connected to a PC with LabView interface. The robot is capable of full 3-degree-of-freedom (DOF) motion (i.e., holonomic $x$-y-translation and $\theta$-rotation) on a planar surface. It is tracked with a long-working-distance video microscope connected to an automated motion capture system.

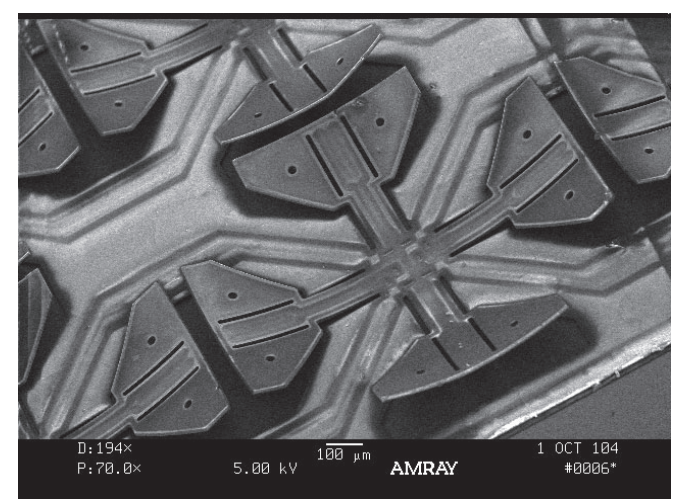

Figure 2. Zoomed-in view of cilia chip fabricated by J. Suh [6] in its unheated state. Resistive heating of a thermo-bimorph cilium decreases its curvature. A "motion pixel" consisting of a group of 4 orthogonal cilia is in the center of the image; independent control of the cilia can generate force or motion in 4 orthogonal directions.

\section{ACTUATOR GAITS}

Unlike insect legs, each of the 512 actuators has only one DOF (increase or decrease of curvature) and thus resembles cilia rather than legs. As a consequence, a single cilium or an array of 
synchronously acting cilia are not useful for inducing motion. However, several effective locomotion schemes using coordinated, out-of-phase motion of the cilia exist and have been investigated.

We define gait in analogy to animal locomotion as a specific sequence of phases, which cause controlled motion when executed by the robot. Fig. 3 depicts the principle of 2-phase, 3-phase, and 4-phase gaits with a single motion pixel. Red (dark) triangles symbolize heated actuators with lower cilium curvature. Transitions from phase \#2 to \#3 and from phase \#3 to \#4 produce steps towards the right, during which the robot body also moves slightly down and up, respectively.

We define gait frequency as the reciprocal of the time to complete one gait cycle. The gait frequency may be different from the frequency at which the cilia activation is changed; this rate is called the step frequency. For example, in a 4-phase gait as shown in Fig. 3 the step frequency is 4 times the gait frequency.

A 3-phase gait leaves out one of the phases above; the better choices for omitted phases are \#1 or \#3. A 2-phase gait uses, e.g., only phases \#3 and \#4. It has been shown to be a less effective gait.

These gaits can be further refined by varying the relative lengths of each phase, with significant effects on power consumption, operating temperature, and robot speed. For example, the power consumption in phase \#1 is 0 , in phase \#2 and \#4 it is $75 \%$, and in phase $\# 3$ it is $100 \%$. If all phases have equal length, then the average power consumption is $1 / 4 \times 0+1 / 2 \times 75 \%+1 / 4 \times 100 \%$ $=61.5 \%$. By increasing the length of phase $\# 1$ and reducing $\# 3$ by 10 percentage points, we can reduce the average power consumption to $52.5 \%$ while maintaining the same gait frequency.

The gait in Fig. 3 does not effectively utilize the cilia $\mathrm{N}$ and $\mathrm{S}$, but they need to be activated during 3 phases so not to obstruct the motion generated by $\mathrm{E}$ and $\mathrm{W}$. Instead, if cilium $\mathrm{N}$ is operated in sync with $\mathrm{E}$, and $\mathrm{S}$ with $\mathrm{W}$, then the robot moves in a diagonal direction towards NE.

Both cilia chips in the robot can be controlled independently, which is useful for rotation. For example, counterclockwise motion is achieved by generating motion towards $\mathrm{N}$ in the right chip and motion towards $\mathrm{S}$ in the left chip, if seen as depicted at the bottom of Fig. 3.

(a)
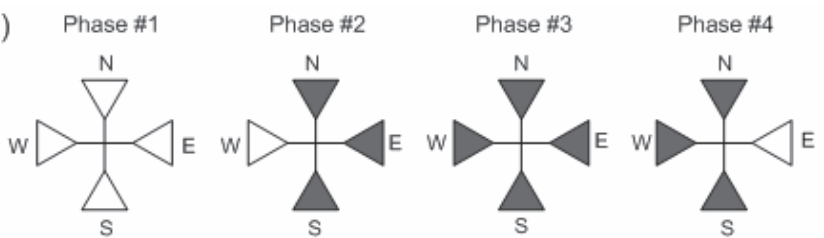

(b)
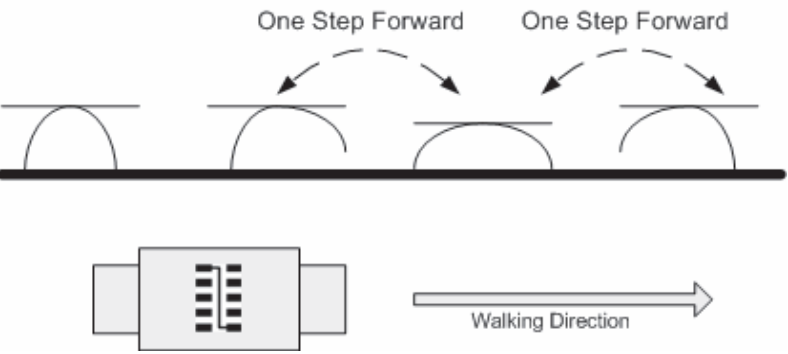

Figure 3. Schematic of a motion pixel executing a 4-phase gait. Red (dark) triangles symbolize heated actuators with lower cilium curvature. Transitions \#2 $\rightarrow \# 3$ and \#3 $\rightarrow$ \#4 produce steps towards the right. A 3-phase gait leaves out one of the phases above, e.g., phase \#1 or \#3. A 2-phase gait uses, e.g., only phases \#3 and \#4.

\section{CHARACTERIZATION OF LOCOMOTION}

Motion of the robot proceeds in discrete steps produced by the actuation gaits (Fig. 3). Step size depends on control parameters including heating power, frequency, duty cycle, but also system parameters such as robot temperature and load. We hypothesize that the robot velocity $v$ follows a function

$$
v(t)=a+b e^{-t / \tau}
$$

where $a+b$ is its initial velocity at room temperature, and $a$ is its steady state velocity when the robot has heated up. $\tau$ describes the time constant of this transition, determined by the robot's thermal mass and heat dissipation. Integrating $v(t)$ leads to an equation for position

$$
x(t)=a t+\tau b\left(1-e^{-t / \tau}\right)
$$

This function has been validated against thermal models using FEM analysis and against large sets of experimental data, as will be described in the following sections.

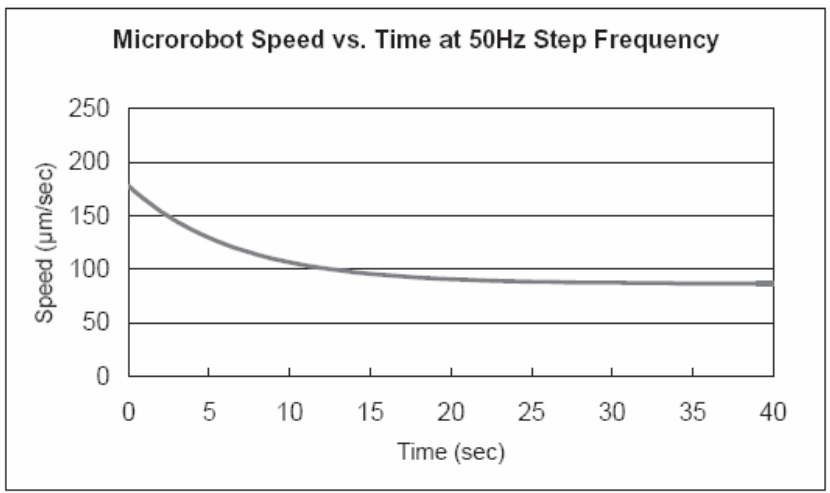

Figure 4. Robot speed at $50 \mathrm{~Hz}$ step frequency $(12.5 \mathrm{~Hz}$ gait frequency). Experimental data (>200 sample points) and curve fitting coincide. $v(0)=178.3 \mu \mathrm{m} / \mathrm{sec}, v(\infty)=86.6 \mu \mathrm{m} / \mathrm{sec}$.

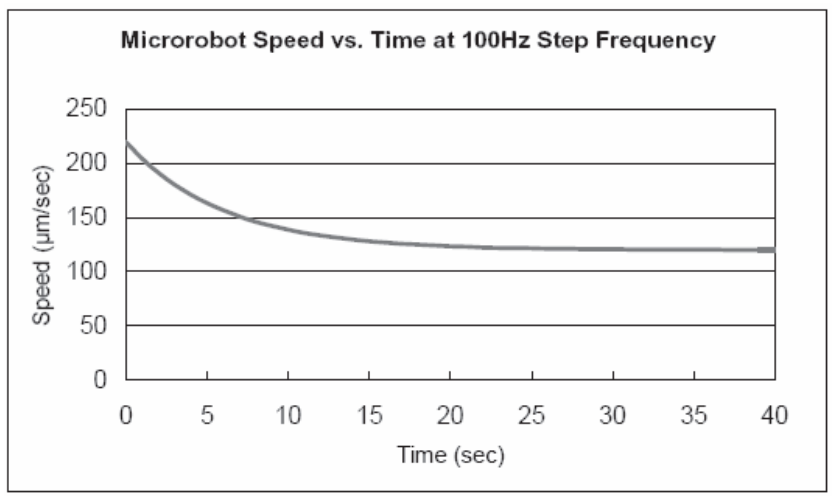

Figure 5. Robot speed at $100 \mathrm{~Hz}$ step frequency $(25 \mathrm{~Hz}$ gait frequency). Experimental data (>200 sample points) and curve fitting coincide. $v(0)=224.4 \mu \mathrm{m} / \mathrm{s}, v(\infty)=124.5 \mu \mathrm{m} / \mathrm{s}$.

\section{WALKING EXPERIMENTS}

The robot is placed onto a flat, level surface with wellknown properties, such as a silicon or glass wafer. The control wires are loosely supported by a stand above the robot such that their weight and stiffness has minimal influence on the robot motion. The control wires are connected to a $60 \mathrm{~V}$ variable power source and switched by a set of 8 power transistors on a custommade controller board linked to a personal computer via a NIDAQ 
interface card. A custom LabView program allows the user to choose parameters such as step and gait frequency, type and direction of gait. An automated tracking system is used to collect motion data from the experiments at the video frame rate.

Figs. 4 and 5 show velocity data for 4 -phase gaits at $12.5 \mathrm{~Hz}$ and $25 \mathrm{~Hz}$ gait frequency over a time span of $40 \mathrm{sec}$. The initial and steady state velocities are $v(0)=178.3 \mu \mathrm{m} / \mathrm{sec}, v(\infty) \approx v(40 \mathrm{sec})=$ $86.6 \mu \mathrm{m} / \mathrm{sec}$ for $12.5 \mathrm{~Hz}$ and $v(0)=224.4 \mu \mathrm{m} / \mathrm{sec}, v(\infty) \approx v(40 \mathrm{sec})=$ $124.5 \mu \mathrm{m} / \mathrm{sec}$ for $25 \mathrm{~Hz}$, respectively. The distance traveled during a single gait cycle can be calculated as $d_{\text {gait }}=v / f_{\text {gait }}$ which is, e.g., approximately $4.5 \mu \mathrm{m}$ at the start of motion with $f_{\text {gait }}=25 \mathrm{~Hz}$.

Fig. 6 summarizes a larger range of such experiments for step frequencies from $50 \mathrm{~Hz}$ to $200 \mathrm{~Hz}$. The slowest speed in steady state is at $50 \mathrm{~Hz}$, and frequencies around 90 to $100 \mathrm{~Hz}$ achieve the highest speeds. Fig. 7 shows the corresponding time constant $\tau$, which was obtained from a least-squares fit between Eq. (2) and the experimental data.

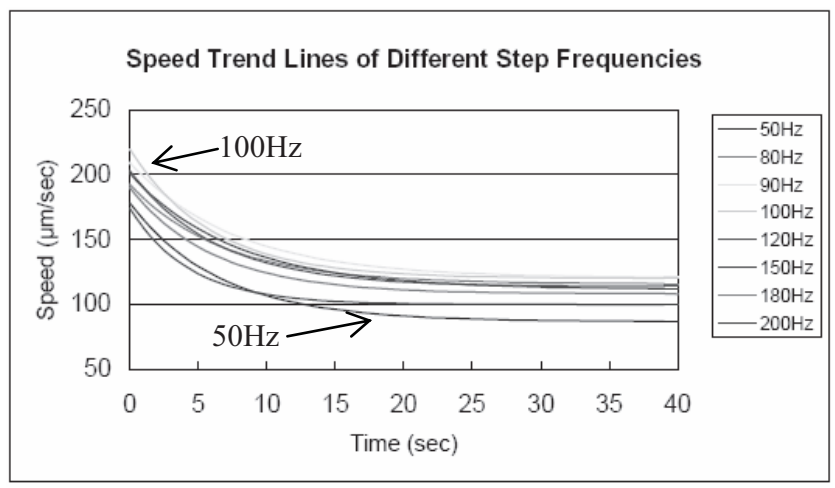

Figure 6. Robot for different step frequencies (experimental data). The absolute values depend on the step frequency and reach a maximum near 90 - 100Hz. The slow-down trend over time is similar in all experiments and reflects the reduced step size as the robot is heating up.

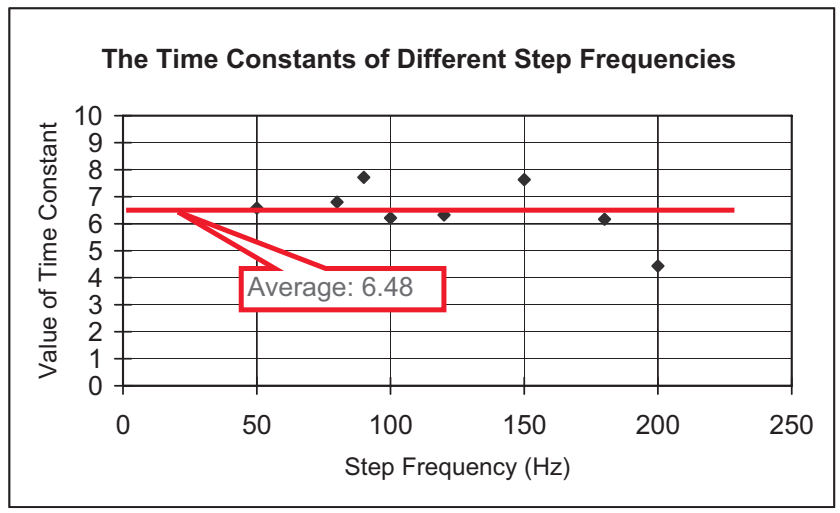

Figure 7. Time constant $\tau$ determined from curve fitting with the experimental data. Similar values are observed for a wide range of control frequencies.

Discussion: From Figs. 4 and 5 we observe that the speed of the robot is higher for the gait frequency of $25 \mathrm{~Hz}$, but it is not twice the speed compared to $12.5 \mathrm{~Hz}$. This implies that the distance traveled during a single gait cycle $d_{\text {gait }}$ decreases with increasing $f_{\text {gait }}$. We believe this decrease is caused by the shorter cooling interval at higher frequencies, which prevents the cilia from reaching their uncooled shape and thus reducing their range of motion. Note, however, that during all of the experiments in Fig. 6 the robot consumes the same amount of electrical power because the duty cycles of the cilia remain unchanged.

\section{MAXIMUM LOAD}

To determine the maximal carrying capacity of the microrobot, additional weight is added until it ceases to move. Fig. 8 shows such an experiment with a heavily loaded robot carrying 4 ICs (2.894gr) and 30 metal staples (0.630gr) in addition to its own mass of $0.478 \mathrm{gr}$ for a total of $4.002 \mathrm{gr}$. This was the largest load observed that the robot was capable of carrying. During a 4-phase gait, 128 cilia must lift this load during the transition from phase $\# 3$ to $\# 4$, corresponding to averagely $306 \mu \mathrm{N}$ per cilium.

Fig. 9 shows an ANSYS FEM simulation of the deflection of a heated cilium with two point loads of $153 \mu \mathrm{N}$ at each corner. The simulation illustrates that the cilium is pressed nearly flat against the substrate under this load.

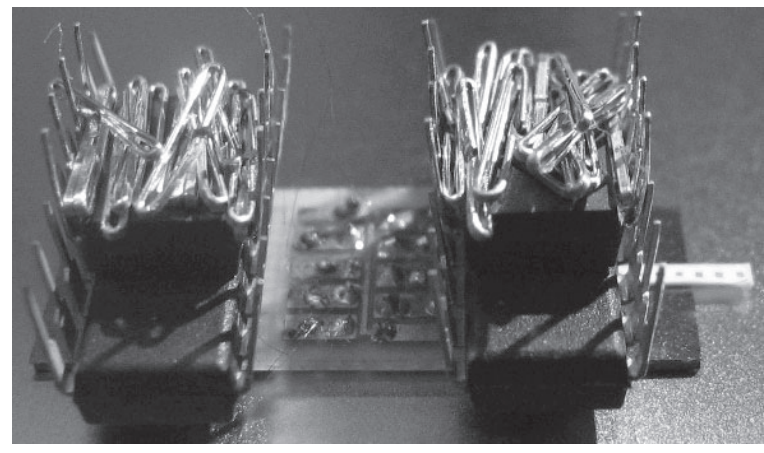

Figure 8. Microrobot walking under maximal load, carrying $3.524 \mathrm{~g}$ in addition to its own mass of $0.478 \mathrm{~g} .128$ cilia lift this load during a 4-phase gait, which implies an average of $31 \mathrm{mg}$ per cilium (more than 1000 times the cilium mass). Note: the small white strip extending over the right edge of the robot holds the patterns that are recognized by the motion tracking system.

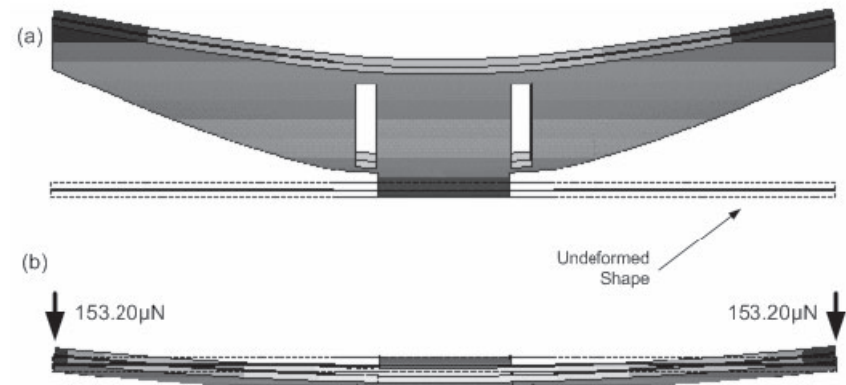

Figure 9. ANSYS FEM simulation of cilium (a) with curvature due to thermal stress, (b) with mechanical load of $153.2 \mu \mathrm{N}$ applied to each corner, corresponding to a mass load of approximately $4 \mathrm{~g}$ distributed over 128 cilia.

\section{SURFACE ROUGHNESS}

To investigate the influence of surface roughness on the robot motion, two sets of experiments are performed: (1) Robot motion on the polished and the unpolished side of a silicon wafer. (2) Robot motion on a polished glass wafer and on a glass wafer roughened by a brief dip in hydrofluoric acid, which produces random features with up to $6 \mu \mathrm{m}$ variation (measured by 
profilometer). In both cases, the step frequency is varied from $100 \mathrm{~Hz}$ to $1000 \mathrm{~Hz}$. We observe that for lower frequencies, the robot speeds are nearly identical for all surfaces. However, for high frequencies, the robot moves slower and with unsteady velocity on the rough surfaces.

Discussion: As stated earlier, the cilia's range of motion decreases at higher frequencies. If the surface roughness becomes significant compared to the cilia range, then the cilia exhibit a tendency to trip or to get trapped.

\section{THERMAL MODELING}

The temperature of the robot body and the walking surface is measured with a small thermocouple (OMEGA HH501BK, 1 sample/sec) by attaching it to the backside of the cilia chip and the backside of the support wafer. Three ANSYS FEM models have been set up to study the thermal characteristics of this system: (1) Macroscopic model of the entire system (consisting of cilia chip, cilia immersed in a boundary layer of air, and support substrate) which describes the overall heat dissipation of the robot. (2) Microscopic model of an individual cilium, which captures thermal stress and strain due to heating and cooling as well as deformations due to applied loads. (3) Fluid solid interaction (FSI) model, which combines structural simulations with computational fluid dynamics to describe the heat transfer between cilia and surrounding air.

Fig. 9 was generated with the "microscopic" model, which recreates a FEM model of the physical cilium shape and materials in great detail. Fig. 10 shows measurements and simulations of the robot temperature in the macroscopic model, which describes cilia chips, support substrate, and air gap as three homogeneous layers.

Discussion: Both the macroscopic and the microscopic model of the robot show excellent agreement with the experimental data. FSI simulation is less successful; so far, no convergence has been achieved between the ANSYS fluid and solid solvers. Likely reasons are the difficulty to accurately model heat conduction and convection in the narrow air gap between cilia chip and support substrate, and the high sensitivity of the cilia to this air flow. A much more detailed discussion of the simulation results can be found in [7].

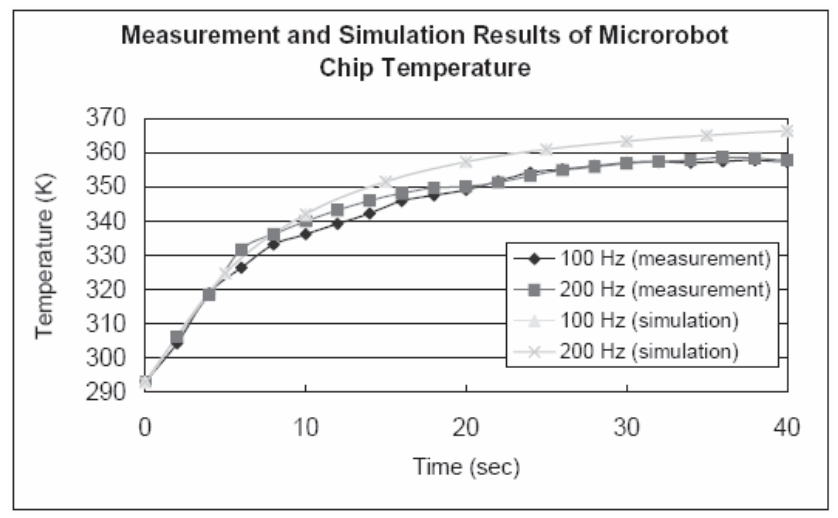

Figure 10. Simulated and measured robot temperatures during operation at $100 \mathrm{~Hz}$ and $200 \mathrm{~Hz}$ step frequency. A "macroscopic" ANSYS FEM model was used to simulate robot body, cilia, walking surface, and air layer in between.

\section{CONCLUSIONS}

A 512-legged walking microrobot capable of full 3-DOF motion across a planar surface has been built and characterized in simulation and experiments. The robot can move at up to $250 \mu \mathrm{m} / \mathrm{sec}$ and carry $3.5 \mathrm{~g}$ load ( $>7$ times its own mass). The robot speed is dependent on its temperature; during operation the robot heats up and slows down accordingly. Its motion can be described by $x(t)=v_{\infty} t+\left(v_{0}-v_{\infty}\right) e^{-t / \tau}$ with $v_{0}$ and $v_{\infty}$ being the robot's initial and steady state velocity (dependent on gait frequency and power supply), respectively, and a thermal time constant $\tau \approx 6.5 \mathrm{sec}$; this model has been validated by FEM analysis and experiments. The robot has proven extremely reliable during operation in noncleanroom environments over many years. A more detailed discussion of the robot is provided in [7].

The robot is strong enough to carry additional components such as small sensors, RF elements, or integrated circuits. For a practical autonomous microrobot, the two most significant challenges are power consumption and coping with rough terrain. More efficient thermo-bimorph cilia [8], piezo actuators [4], or electrostatic actuators powered by solar cells [3] are examples to address the first challenge. The small range of cilia motion and the rigid robot body limit the current robot's operation to flat surfaces that are rarely found in uncontrolled environments. Larger range of actuator motion and body compliance would make the robot more widely applicable to rough terrain.

\section{ACKNOWLEDGEMENTS}

The authors would like to thank Eric Black, Matt Mohebbi, Anshuman Shukla, Kerwin Wang, Xiaorong Xiong and all the members of the UW MEMS laboratory for their help on this project, and all the staff of the Stanford Nanofabrication Facility (SNF) for their professional and dedicated service. Support for the ciliary array chip research was initially supported by DARPA under contract N001-92-J-1940-P00001 and two additional years were provided by a General Motors Key grant and Kovacs NSF NYI award (ECS-9358289-006). Further support was provided to K. Böhringer by NSF CAREER award ECS-9875367 and by a JSPS fellowship in 2004-2005.

\section{REFERENCES}

1. Takeshima, N. and H. Fujita. Polyimide bimorph actuators for a ciliary motion system, in ASME WAM, Symp. Micromech. Sensors, Actuators, and Systems. 1991.

2. Ebefors, T., J.U. Mattsson, E. Kèlvesten, and G. Stemme. A robust micro conveyor realized by arrayed polyimide joint actuators, in IEEE 12th Workshop on Micro Electro Mechanical Systems (MEMS). 1999. Orlando, FL.

3. Hollar, S., A. Flynn, C. Bellew, K.S.J. Pister. Solar Powered 10mg Silicon Robot, in IEEE MEMS. 2003. Kyoto, Japan.

4. Suhr, S.H., S.J. Lee, Y.S. Song, and M. Sitti. Biologically Inspired Miniature Water Strider Robot, in Robotics: Science and Systems I. 2005. Cambridge, MA.

5. Mohebbi, M.H., M.L. Terry, K.F. Böhringer, J.W. Suh, and G.T.A. Kovacs. Omnidirectional Walking Microrobot Using MEMS Thermal Cilia Arrays, in ASME International Mechanical Engineering Congress and Exposition (IMECE'01). 2001. New York, NY.

6. Suh, J.W., S.F. Glander, R.B. Darling, C.W. Storment, and G.T.A. Kovacs, Organic thermal and electrostatic ciliary microactuator array for object manipulation. Sensors and Actuators, A: Physical, 1997. 58:51-60.

7. Chen, Y.-M., Thermal Model of Microrobot, Masters Thesis, Electrical Engineering. 2005, University of Washington, Seattle, WA.

8. Suh, J.W., R.B. Darling, K.F. Böhringer, B.R. Donald, H. Baltes, and G.T.A. Kovacs, CMOS integrated ciliary actuator array as a general-purpose micromanipulation tool for small objects. ASME/IEEE Journal of Microelectromechanical Systems, 1999. 8(4):483-496. 